\title{
Factores predictivos de mortalidad y de riesgo de ingreso en cuidados intensivos de los pacientes hospitalizados infectados por Sars-cov-2 en el área sanitaria Galicia sur
}

\author{
Predictive factors of mortality and risk of admission in a critical care unit \\ of hospitalized patients infected by Sars-cov-2 in the sanitary area Galicia sur
}

\author{
María Luz Cánovas Martínez', Noelia Gago Diéguez', Olalla Figueiredo Gonzalez', Nuria Adán Valencia', Eva Villar \\ Arcay', Ariadna Rodríguez Rodríguez', Raquel Ruido Dacal', Cristina Martínez Reglero² \\ ${ }^{1}$ Servicio de Anestesia Reanimación y Dolor del Complexo Hospitalario Universitario Ourense. ${ }^{2}$ Unidad de Metodología y Estadística. Instituto de Investigación Sanitaria \\ Galicia Sur
}

\section{RESUMEN}

Objetivo: Determinar factores de riesgo de gravedad y mortalidad en pacientes ingresados por COVID 19 en la población de Ourense.

Método: Estudio prospectivo en 342 pacientes hospitalizados en el Hospital Universitario de Ourense por COVID 19 entre el 8 de marzo y 15 de mayo del 2020, periodo correspondiente a la primera ola de contagios.

Se recogieron variables demográficas, patologías y tratamientos previos, analítica y tratamientos durante el ingreso. Se relacionaron con el riesgo de ingreso en cuidados intensivos y con mortalidad.

Se llevó a cabo un análisis univariante y uno multivariante de regresión logísitca. Para la comparación de variables cualitativas se utilizó la prueba Chi-cuadrado. Para la comparación de las variables cuantitativas, se analizó la normalidad de la distribución de los datos en cada uno de los grupos muestrales y se aplicó el test t-Student o Mann-Whitney. El nivel de significación aceptado para todos los contrastes de hipótesis fue de 0,05. Resultados y Conclusiones: Edad $>75$, linfocitos $<0.9$, Dímero $D>2500$ se correlacionaron positivamente con la mortalidad. Obesidad, linfocitos $<0$.8, Dímero $D>2500, \mathrm{LDH}>500$ y ferritina $>800$ se correlacionaron positivamente con el riesgo de ingreso en cuidados intensivos. Tratamiento con Lopinavir/Ritonavir, Hidroxicloroquina, corticoides y anticoagulantes, actuaron como factores protectores frente a la mortalidad.

Palabras clave: COVID; críticos; mortalidad; tratamiento; patologías

\section{INTRODUCCIÓN}

En diciembre de 2019, se identificaron varios casos de neumonía de origen desconocido en Wuhan, Hubei, China. El patógeno fue identificado como nuevo coronavirus (CoV) perteneciente al género b-CoV. El cuadro respiratorio que produce fue rebautizado como Síndrome Respiratorio Agudo Grave (SARS) CoV-2 (SARS-CoV-2, anteriormente llamado en 2019 nuevo CoV). Este nuevo virus comparte un $87,99 \%$ de identidad de secuencia con el SARS de murciélago, como CoV y el 79,5\% de su secuencia con SARS-CoV como publican Caizheng et al ${ }^{1}$, Trilla $\mathrm{A}^{2}$ y Shao et $\mathrm{a}^{3}$.

A fecha de agosto del 2020, el número de casos confirmados en el mundo ascendió a 18.614.177, de estos 3.245.365 casos confirmados en Europa y 314.362 en España. En la Comunidad Gallega, en esta misma fecha, el número total de casos confirmados ascendió a 12.640, con 11.428 casos curados, 591 activos y una mortalidad del 4,9\%. La provincia de Ourense, según la Dirección General de Salud Pública de la Consellería de Sanidad de la Xunta de Galicial ${ }^{4}$, al

\section{ABSTRACT}

Objective: To determine severity and mortality risk factors in patients admitted to hospital for COVID 19 in the population of Ourense.

Method: Prospective study in 342 patients hospitalized at the University Hospital of Ourense for COVID 19 between March 8 and May 15, 2020, the period corresponding to the first wave of infections.

Demographic variables, pathologies and previous treatments, laboratory tests and treatments during admission were collected and were related to the risk of admission to critical patients and mortality.

A univariate analysis and a multivariate logistic regression analysis were carried out. For the comparison of qualitative variables, the Chi-square test was used. For the comparison of the quantitative variables, the normality of the distribution of the data in each of the sample groups was analyzed and the t-Student or Mann-Whitney test was applied. The accepted level of significance for all hypothesis tests was 0.05 .

Results and Conclusions: Age $>75$, lymphocytes $<0.9$, D-dimer $>2500$ were positively correlated with mortality. Obesity, lymphocytes $<0.8$, Ddimer> 2500, LDH> 500 and ferritin> 800 were positively correlated with the risk of admission to critical patients. Treatment with Lopinavir/ Ritonavir, Hydroxychloroquine, corticosteroids, and anticoagulants acted as protective factors against mortality.

Key Words: COVID; critical; mortality; treatment; pathology.

final de la primera ola, se registraron un total de 2012 casos, con más de 300 ingresos que supuso aproximadamente un $17 \%$ de los infectados y una mortalidad global del 6,56\%.

Basándonos en el interés científico por conocer que condicionantes o características de los individuos pueden aumentar o disminuir el riesgo de padecer COVID- 19 en nuestra provincia hemos planteado como objetivos de este estudio:

- Analizar datos demográficos, comorbilidad, tratamientos previos y datos analíticos de los pacientes ingresados en el Hospital Universitario de Ourense, diagnosticados de COVID-19 para tratar de establecer variables predictoras de mortalidad y de ingreso en unidades de críticos.

- Hacer una estimación de aquellas variables que actuarían como factor protector o factor de riesgo, tanto de mortalidad como de ingreso en críticos y en que medida.

- Correlacionar dichas variables con la gravedad y la mortalidad. 


\section{MATERIAL Y MÉTODO}

Se realizó un estudio prospectivo en 342 pacientes durante el periodo comprendido entre el 8 de marzo, fecha de hospitalización del primer paciente ingresado en el Hospital Universitario Ourense con este diagnóstico y el 15 de mayo del 2020, fecha en la que ingresó el último paciente antes del comienzo de la segunda ola de contagios. El estudio fue aprobado por el comité ético de investigación clínica del Centro.

La variables estudiadas fueron:

- Datos demográficos: Edad; Sexo; Fecha de comienzo de Ios síntomas; Fecha de ingreso hospitalario; Ingreso en UCl y días de ingreso.

- Patologías previas: hipertensión arterial (HTA); dolor crónico; diabetes; obesidad (índice de masa corporal, IMC $30 \mathrm{Kg} /$ $\mathrm{m}^{2}$ ); dislipemia; hiperuricemia; infarto agudo de miocardio (IAM); fibrilación auricular(FA); insuficiencia cardíaca (IC); otros antecedentes cardíacos (AC); tromboembolismo pulmonar (TEP); ictus; enfermedad pulmonar obstructiva crónica (EPOC); asma; historia de cáncer previo y actual e insuficiencia renal crónica (IRC).

- Tratamientos previos: inhibidores de la enzima convertidora de angiotensina (IECA); bloqueadores de los receptores de la angiotensina II (ARAll); inhibidores de la aldosterona; antagonistas del calcio (ACA); diuréticos; anticoagulantes (ACO); antiagregantes; antiinflamatorios no esteroideos (AINES); corticoides; paracetamol; metamizol; estatinas; antidiabéticos orales (ADO); insulina; tramadol; tapentadol; oxicodona/naloxona; fentanilo; buprenorfina; anticonvulsivantes; antidepresivos (ADT); lidocaína intravenosa (i.v.); vitamina D; hidromorfona; ketamina; canabinoides (THC); biológicos; fibratos; quimioterapia (QT); radioterapia (RT); trasplante y tratamiento inmunosupresor.

- Datos de laboratorio: ferritina; dímero D; LDH y linfocitos.

- Tratamientos recibidos para la COVID-19: lopinavir/ritonavir; hidroxicloroquina; azitromicina; remdesivir; tocilizumab; corticoides; anticoagulantes; otros tratamientos (interferón y otros antibióticos)

- Soporte Respiratorio: oxígeno por gafas nasales; ventimask; Monaghan (ventilación con reservorio); ventilación mecánica (días) y ECMO (oxigenación por membrana extracorpórea).

- Prono.

- Desenlace: alta; éxitus.

Estudio Estadístico: Se realizó un análisis descriptivo de los datos. Las variables cualitativas se expresaron en número $\left(n^{0}\right)$ y porcentaje (\%). Las variables cuantitativas se presentaron con su media y desviación estándar (DE) o mediana y rangos intercuartílicos (RI) si no se ajustaban a una distribución normal. Se utilizó la prueba de Kolomogorov-Smirnov para contrastar si las variables se distribuían normalmente.

Se llevó a cabo un análisis univariante para determinar que variables podrían presentar efecto independiente sobre el ingreso en críticos y la mortalidad hospitalaria. Para la comparación de dos variables cualitativas se utilizó la prueba Chi-cuadrado. Para la comparación de las variables cuantitativas, se analizará la normalidad de la distribución de los datos en cada uno de los grupos muestrales y se aplicará el test t-Student o Mann-Whitney.

Posteriormente, incluyendo como variables independientes a aquellas que mostraron en el análisis univariante alguna relación con el ingreso en críticos o la mortalidad se realizó una regresión logística multivariante, analizando los posibles factores de confusión e interacción y la colinealidad entre las variables.

El nivel de significación aceptado para todos los contrastes de hipótesis fue de 0,05. Los datos fueron analizados con el Software SPSS versión 19.

Este estudio forma parte del estudio multicéntrico nacional con código VMA-BUP-2020-01.

\section{RESULTADOS}

La edad media de los pacientes que ingresaron durante este periodo fue 69,83 17,01 años, 189 (55\%) fueron hombres y 154 (45\%) mujeres. La edad de las mujeres fue significativamente superior a la de los hombres $(79,2 \pm 11,5$ frente a $69,4 \pm 14,5 ; p<0,05) ; 30$ pacientes $(8,7 \%)$ fueron ingresados en cuidados intensivos. La mortalidad global fue del 16,1\% ( $n=55)$. Las características sociodemográficas y analíticas de supervivientes, fallecidos, ingresados y no ingresados en críticos se representan en la Tabla I. Un total de 288 fueron dados de alta $(82,2 \%)$. El tiempo desde el inicio de los síntomas hasta el ingreso hospitalario fue de 10,7 días con un rango ( $r$ ) entre 1 y 13 días. La estancia media en el hospital previa al alta fue 12,4 días (r:1-67).

Los patologías previas más frecuentes fueron: obesidad en 221 pacientes (64,6\%), HTA en $172(50,3 \%$ ) y dislipemia en 121 (35,4\%). 81 pacientes (23,8 \%) padecían dolor crónico. El tipo de dolor más frecuente fue Somático (10,2\%), Mixto $(7,6 \%)$, Neuropático (5\%) y Visceral $(0,9 \%)$.

Los tratamientos previos al ingreso, que tomaban estos pacientes, se representan en la Tabla II, siendo los más frecuentes estatinas, antihipertensivos (diuréticos, ARA II, IECA, ACA e inhibidores de la aldosterona) y los antiagregantes. Los fármacos más utilizados en el tratamiento del dolor fueron los adyuvantes, entre los que destacan antidepresivos tricíclicos (ADT) en 57 pacientes (16,6\%) y anticonvulsivantes en $45(13,5 \%)$, seguidos del paracetamol en $50(0,6 \%)$. Los tratamientos de la COVID- 19 recibidos durante el ingreso se representan en la Tabla III.

Con respecto al soporte de oxígeno: 29 pacientes $(8,47 \%)$ precisaron soporte ventilatorio mecánico en unidades de cuidados intensivos; $18(5,3 \%)$ recibieron oxígeno a través de Monasghan/reservorio; 13 (3,8\%) utilizaron ventimask a 
Tabla I. Variables sociodemográficas y analíticas

\begin{tabular}{|l|c|c|c|c|c|c|}
\hline & Supervivientes & Fallecidos & $p$ & UCl & Planta & $p$ \\
\hline $\mathrm{n}$ & 287 & 55 & $p>0,01$ & 30 & 312 & \\
Edad Media $\pm \mathrm{DE}$ & $67,88 \pm 17,4$ & $88,1 \pm 11,5$ & $<0,001$ & $62,19 \pm 11.97$ & $70,9 \pm 17,6$ & 0,06 \\
Sexo: & & & & & \\
Mujeres & $128(83,9 \%)$ & $25(16,4 \%)$ & $p=0,0882$ & $9(5,8 \%)$ & $144(94,1 \%)$ & $p=0,060$ \\
Hombres & $159(80,9 \%)$ & $30(15,8 \%)$ & & $21(11,7 \%)$ & $168(88 \%)$ & \\
Éxitus & & & & $6(20 \%)$ & $49(15,7 \%)$ & $p=0,653$ \\
Analítica & & & & & \\
Ferritina (ng/ml) & $703,84 \pm 889,962$ & $999,24 \pm 1429,73$ & 0,03 & $1670,3 \pm 1856,2$ & $656,5 \pm 795,6$ & $<0,001$ \\
Linfocitos (miles/mm3) & $1,37 \pm 1,79$ & $0,82 \pm 0,51$ & 0,001 & $0 ., 9 \pm 0,33$ & $1,32 \pm 1,72$ & 0,01 \\
Dímero D (microgr/ml) & $2075 \pm 6230$ & $3642,7 \pm 5434,4$ & 0,004 & $10680,3 \pm 18833$ & $1654 \pm 3014$ & 0,001 \\
LDH (U/l) & $555,28 \pm 266,03$ & $683,9 \pm 297,261$ & 0.21 & $922.94 \pm 357.78$ & $546.46 \pm 249.52$ & 0.04 \\
\hline
\end{tabular}

UCl: Unidad de cuidados intensivos, DE: desviación estandart

diferentes concentraciones; 236 (69\%) recibieron oxígeno a diferentes concentraciones a través de gafas nasales y $23(6,8 \%)$ no precisaron aporte de oxígeno. 40 $(11,7 \%)$ de los pacientes precisaron ser pronados de los que 27 (67,5\%) se encontraban con ventilación mecánica en unidades de cuidados intensivos.

Factores relacionados con ingreso en unidades de cuidados intensivos

El $11,7 \%$ de los hombres y el $5,8 \%$ de las mujeres fueron ingresadas en unidades de cuidados intensivos. Sin embargo el ser mujer redujo en un 5,32 \% el riesgo de ingreso, actuando como factor protector (OR 0,468. IC 95\%: 0,209-1,050. $p=0,060$ ).

La edad media de los pacientes que ingresaron en cuidados intensivos fue significativamente inferior a la de aquellos que no ( $\mathrm{p}=0,001)$ (Tabla I).

No existió relación significativa entre las patología previas que presentaban los pacientes y el ingreso en cuidados intensivos. Aunque patologías como la obesidad (OR odds ratio: 2. IC 95\%: 0,822-5,035. $p=0,016$ ), dislipemia (OR 1,35. IC 95\%: 0,644-2,818. $p=0,23$ ), IC (OR 1,85. IC 95\%: 0,152-6,712. $p=0,409$ ) y asma (OR 2,56. IC 95\%: 0,805-8,159. $p=0,11$ ) se relacionaron con el riesgo de ingreso en cuidados intensivos aunque sin significación estadística ( $p>0.05)$.

Con respecto a los tratamientos previos que tomaban los pacientes, existió relación significativa entre el ingreso en cuidados intensivos y el tratamiento con IECA (OR 4.52. IC95\% 1,545-13,255. $\mathrm{p}=0,003)$ ). Lo mismo sucede con los ARA II (OR 4,14. IC 95\% 1,508-12,932. $p=0,003$ ), inhibidores de la aldosterona ( OR 4,52. IC $95 \% 1,7-12,13$. $p=0,001)$, ACA (OR 4,59. IC95\% 1,5712,23. $p=0,001)$.

El resto de los tratamientos: diuréticos, antiagregantes, anticoagulantes analgésicos, antidiabéticos orales,
Tabla II. Tratamientos previos al ingreso

\begin{tabular}{|l|ll|lc|}
\hline Tratamientos previos & \multicolumn{2}{|c|}{ Si } & \multicolumn{2}{c|}{ No } \\
\hline & $n$ & $\%$ & $n$ & $\%$ \\
\hline IECA & 209 & 62,4 & 126 & 37,6 \\
ARA II & 215 & 62,9 & 127 & 37,1 \\
Inhibidores aldoterona & 192 & 56,1 & 150 & 43,9 \\
ACA & 212 & 62,0 & 130 & 38,0 \\
Diuréticos & 231 & 67,5 & 110 & 32,2 \\
ACO & 213 & 62,3 & 129 & 37,7 \\
Antiagregantes & 228 & 66,7 & 114 & 33,5 \\
Corticoides & 185 & 54,1 & 156 & 45,6 \\
Estatinas & 244 & 71,3 & 97 & 28,4 \\
ADO & 205 & 59,9 & 134 & 39,2 \\
Insulina & 192 & 56,1 & 150 & 43,9 \\
Biológicos* & 3 & 0,87 & 339 & 99,1 \\
Fibratos & 192 & 56,1 & 150 & 43,9 \\
\hline \multicolumn{2}{|c}{${ }^{* B i i o l o ́ g i c o s: ~ a n t i c u e r p o s ~ m o n o c l o n a l e s, ~ t r a s t u z u m a b, ~ p e r t u z u m a b ~}$}
\end{tabular}

Tabla III. Tratamientos recibidos durante el ingreso

\begin{tabular}{|l|ll|lc|}
\hline Tratamientos previos & \multicolumn{2}{|c|}{ Si } & \multicolumn{2}{c|}{ No } \\
\hline & $n$ & $\%$ & $n$ & $\%$ \\
\hline Lopinavir/ritonavir & 140 & 40,9 & 201 & 58,8 \\
Hidroxicloroquina & 286 & 83,6 & 56 & 16,4 \\
Azitromicina & 276 & 80,7 & 66 & 19,3 \\
Tocilizumab $^{\text {Remdesivir }}{ }^{1}$ & 13 & 3,8 & 329 & 96,2 \\
Corticoides $^{\text {Anticoagulantes }}$ & 1 & 0,3 & 341 & 99,7 \\
Otros tratamientos $^{2}$ & 92 & 26,9 & 250 & 73,1 \\
Otros antibióticos $^{2}$ & 49 & 14,3 & 293 & 85,7 \\
\hline
\end{tabular}

${ }^{1}$ Paciente ingresado en unidad de críticos. ${ }^{2}$ aciclovir, oseltamivir y tipos de interferón 
Tabla IV: Análisis Multivariante de Regresión Logística de los factores de riesgo de ingreso en UCI para pacientes Covid-19

\begin{tabular}{|l|c|c|c|c|}
\hline Variables & B & Wald & OR (IC95\%) & $p$ \\
\hline Edad $>65$ & $-0,58$ & 11,77 & $0,94(0,91-0,97)$ & 0,01 \\
Consumo de antiagregantes & 3,96 & 10,322 & $0,096(0,01-0,023)$ & 0,042 \\
Inhib aldosterona & $-2,34$ & 4,117 & $52,52(4,68-588,61)$ & 0,001 \\
DD $>$ 2000mcgr/ml & 0,038 & 20,35 & $1,002(1,001-1,003)$ & 0,000 \\
LDH $>650 U$ ( & 0,002 & 18,23 & $1,039(1,022-1,058)$ & 0,000 \\
\hline
\end{tabular}

Tabla V: Análisis Multivariante de Regresión Logística de los factores de riesgo de mortalidad para pacientes Covid-19

\begin{tabular}{|l|c|c|c|c|}
\hline Variables & B & Wald & OR (IC95\%) & $p$ \\
\hline Edad >75 años & 0,055 & 16,62 & $1,057(1,029-1,088)$ & 0,014 \\
LDH (UI) & 0,013 & 5,97 & $1,013(1,003-1,024$ & 0,000 \\
Corticoides & 1,385 & 15,36 & $3,998(1,999-7,090)$ & 0,006 \\
Hidroxicloroquina & $-1,184$ & 7,15 & $0,306(0,13-0,713)$ & 0,000 \\
\hline
\end{tabular}

insulina, estatinas, vitamina D, fibratos, quimioterapia, radioterapia, inmunosupresores y la condición de trasplantado no influyeron significativamente en el ingreso en cuidados intensivos.

Los pacientes que ingresaron en cuidados intensivos presentaron niveles significativamente mayores de ferritina, dímero $D$ y LDH que los que fueron tratados en planta. La linfopenia fue significativamente más severa en los pacientes que ingresaron en cuidados intensivos $p<0,001$ (Tabla I). Por lo tanto niveles elevados de ferritina, dímero D, LDH Y la linfopenia se relacionaron con el riesgo de ingreso en cuidados intensivos.

El tratamiento con Lopinavir/ritonavir $(p=0,027)$, Hidroxicloroquina $(0,038)$, Tocilizumab $(p<0,001)$, corticoides $(p<0,001)$, anticoagulantes $(p=0,001)$ y otros antibióticos diferentes a la azitromicina $(p=0,006)$ se relacionaron con un mayor grado de ingresos en cuidados intensivos.

En el análisis multivariante se observó que el riesgo de ingreso en cuidados intensivos disminuye a medida que aumenta la edad del paciente y en aquellos que toman antiagregantes. Sin embargo aumenta con el consumo de inhibidores de la aldosterona y niveles más altos de LDH y DD (Tabla IV).

\section{Factores asociados a la mortalidad}

55 (16.11\%) pacientes fallecieron. 30 hombres $(15,8 \%)$ frente a 25 mujeres $(16,4 \%)$ por lo que no existieron diferencias significativas en la mortalidad para cada sexo $(p=0,882)$.

La edad media de los pacientes que sobrevivieron fue significativamente menor que la de los éxitus $(p<0,001)$ (Tabla I).

Patologías como la HTA $(p=0,096)$, dolor crónico $(p=0,34)$, diabetes $(p=0,1)$, obesidad $p=0,103)$, dislipemia $(p=0,063)$, hiperuricemia $(p=0,601)$, IAM $(p=0,052)$, IC $(p=0,06)$, FA $(p=0,08)$, TEP/TVP $(p=0,394)$, asma $(p=0,753)$, cáncer previo $(p=0,601) 0$ actual $(p=0,295)$ no influyeron significativamente en la mortalidad. Otras patologías como: otros an- tecedentes cardíacos (OR 2.140. IC 95\% 0,148-5,515. $p=$ 0,047); historia de ictus (OR 2.870. IC95\% 1,305-6,308. $p=0,07)$; EPOC (OR 3,160. IC 95\% 1,304-7,398. $p=0,021)$ e IRC (OR 4,893. IC 95\% 1,576-15,188. $p=0,009)$ se relacionaron con el riesgo de muerte por COVID-19.

El tratamiento con fármacos antihipertensivos, anticoagulantes y antiagregantes, analgésicos de los tres niveles de la escalera analgésica de la OMS, hipolipemiantes, quimioterapia y radioterapia así como los fármacos inmunosupresores no incrementaron el riesgo de mortalidad de forma significativa $(p>0,05)$.

En relación a los datos de laboratorio no hubo diferencias significativas en los niveles medios de ferritina entre los pacientes que fueron alta y los fallecidos. Sin embargo los niveles de linfocitos fueron significativamente más bajos en los éxitus que en las altas y las determinaciones de dímero D y LDH significativamente más altas en los éxitus.

$49(15,9 \%)$ de los pacientes ingresados en planta y 6 $(19,2 \%)$ de los ingresados en una unidad cuidados intensivos fueron éxitus. El ingreso en una unidad de cuidados intensivos no fue factor de riesgo de mortalidad $p=0,653$.

Analizando los tratamiento que recibieron los pacientes durante el ingreso, el tratamiento con lopinavir/ritonavir (OR 4,53. IC 95\%0,236-0,871.p=0,016); hidroxicloroquina ( $\mathrm{OR}=$ 0,371 . IC95\% 0,189-7,31. $p=0,003$ ) actuaron como factores protectores frente a la mortalidad de forma significativa.

El tratamiento con corticoides (OR 3,280. IC95\% 1,7935,999. $p=0,003$ ); anticoagulantes (OR 2,076. IC95\% $0,995-4,328 . p=0,048)$ y otros antibióticos diferentes a la azitromicina (OR 2,452. IC95\% 1,109-5,999. $p=0,023$ ) incrementaron el riesgo de mortalidad de forma significativa. Azitromicina (OR 0,776 IC95\% 0,382-1,578. $p=0,483$ ) y remdesivir (OR 0,171. IC95\% 0,121-0,243. $\mathrm{p}=0 ., 76$ ) actuaron como factores protectores frente a la mortalidad sin significación estadística. 
El tratamiento con tocilizumab (OR 1,162. IC95\% 0,2445,535. $p=0,693$ ) actuó como factor de riesgo de mortalidad pero sin significación estadística.

El 17,4\% de los pacientes que recibieron prono fueron éxitus, frente al $16,6 \%$ de los que no estuvieron en prono ( $p=0,774)$.

En el análisis multivariante se observó que la edad $>75$ años, el aumento de la LDH y el consumo de corticoides se relacionó con un aumento de mortalidad. Sin embargo el tratamiento con hidroxicloroquina se relacionó con una disminución del riesgo de mortalidad (Tabla V).

\section{DISCUSIÓN}

En este estudio analizamos una gran serie de pacientes hospitalizados por COVID-19 incluidos en el estudio observacional y multicéntrico código VMA-BJUP-2020-01. Incluyó pacientes ingresados en el EOXI de Ourense de forma consecutiva, procedentes de su domicilio, residencias de ancianos y otros centros hospitalarios. Al igual que en otros estudios, como el de Li Tan et al. ${ }^{5}$ nuestros pacientes fueron predominantemente varones, de edad avanzada, y con varias comorbilidades.

En nuestra serie la edad mediana de los pacientes es más alta que la de otros estudios, como el de Caizheng et al ${ }^{1}$, hecho que se pueda atribuir a que nuestra población es de las más envejecidas de Galicia. La mayor edad va acompañada de una mayor morbilidad: obesidad, HTA y dislipemia son las patologías más frecuentes en nuestros pacientes, resultados similares al estudio de Richardson et al ${ }^{6}$.

El porcentaje de pacientes que ingresaron en unidades de cuidados intensivos es similar al de otros estudios multicéntricos nacionales, como el de Casas-Rojo et al. ${ }^{7}$ y menor al porcentaje de ingreso de otros estudios internacionales como el de Zhou et al|, y el de Docherty AB et al ${ }^{9}$. Con respecto al tiempo de estancia en una unidad de cuidados intensivos, el tiempo de estancia de nuestros pacientes fue superior al publicado por otros estudios como el de Young et al..$^{10} \mathrm{y}$ el de Kuwaski et al ${ }^{11}$. de 7 días para los supervivientes y para los no supervivientes de 8 días. Este hecho se puede explcar debido a que la mayoría de nuestros pacientes ingresados en cuidados intensivos precisaron soporte ventilatorio y más del 80\% fueron pronados.

En nuestro estudio se mantienen algunos de los factores descritos en la literatura como predictores de peor pronóstico en la infección por SARS-CoV-2 como es el sexo masculino resultados que coinciden con los de Marta et a $\left.\right|^{12}$ y Wang et al. ${ }^{13}$. La edad de los que ingresaron en cuidados intensivos fue menor de la de aquellos que permanecieron en la planta. Este hecho podría explicar la no relación entre las patologías previas y el pronóstico de los mismos, resultados que coinciden con los de otros estudios como el de Ferrando et a $\left.\right|^{14}$.Otro factor que podría influir es que aquellos pacientes con antecedentes de patologías graves, de edad avanzada que desarrollaron un cuadro clínico severo no fueron candidatos a UCl. Sin embargo hay un grupo de personas jóvenes y sin patologías previas conocidas en las cuales la infección por SAS-Cov-2 produce una reacción inflamatoria severa provocando la necesidad de hospitalización en cuidados intensivos ${ }^{15}$.

Cheng et al ${ }^{16}$ ha observado que los casos de evolución grave presentaban un aumento de parámetros inflamatorios y citopenias leves. En nuestra población, los niveles elevados de dímero-D y LDH se asociaron con una mayor severidad de la enfermedad. En pacientes con enfermedad pulmonar intersticial severa, el aumento de LDH fue significativo y es uno de los marcadores pronóstico más importantes de lesión pulmonar. Para los pacientes críticamente enfermos con COVID-19, el aumento del nivel de LDH va a indicar un aumento de la actividad y extensión de la lesión pulmonar.

La linfopenia es una característica común en nuestros pacientes pero no parece ser un factor crítico asociado con la gravedad de la enfermedad. Las células epiteliales alveolares lesionadas podrían inducir la infiltración de linfocitos, lo que lleva a una linfopenia persistente que se observa en los pacientes como en el estudio de Yang et $a^{5}$. Niveles altos de ferritina, LDH, dímero $D$ y linfopenia son considerados marcadores de gravedad por otros estudios como el de Casas et al:; Marta et $\mathrm{a}^{12} ; \mathrm{y}$ Cheng et $\mathrm{al}^{16}$.

La mortalidad global en nuestros estudio fue superior a la publicada por la Dirección General de Salud Pública de la Consellería 4 hecho que se explica porque los pacientes ingresados presentaban más factores de riesgo y mayor gravedad, que la población general Covid-19 positiva de Ourense.

Llama la atención la diferencia de mortalidad entre nuestros pacientes y el estudio Onder et al. ${ }^{17}$ teniendo en cuenta que compartimos características demográficas, además de que el desarrollo en el tiempo de la pandemia y su magnitud ha sido casi similar en Italia con respecto a España. Esta diferencia en la mortalidad puede deberse a unos criterios de ingreso hospitalario menos estrictos lo que lleva consigo un mayor número de ingresos más leves en relación con los nuestros. Otros estudios publican unos índices de mortalidad por encima de los nuestros, como los de Yang et al ${ }^{5}$; Richardson et al ${ }^{6}$ y Zhou et al $\left.\right|^{8}$.

El sexo no influyó en la mortalidad en comparación con otros estudios como el COVID 19 in EU/EEA and the UK $2020^{18}$ que publican una proporción del doble de muertes de hombres frente a mujeres, este resultado podría ser debido a que la edad media de las mujeres ingresadas superaba a la de los varones.

Edad avanzada, antecedentes de ictus, IRC, EPOC y antecedentes cardíacos diferentes al IAM, IC o FA, incrementaron el riesgo de mortalidad. Algunas de estas patologías coinciden con las de otras publicaciones como la de Casas- Rojo et al. ${ }^{7}$ y la publicada por el Chinese Center for disease Control and Prevention ${ }^{19}$, aunque diferentes grupos de pacientes pueden presentar distintos factores desfavorables de desenlace fatal como los publicado por Shang et al20.

Edad avanzada, aumento de LDH y consumo de corticoides se correlacionaron, en nuestro estudio, con el riesgo de muerte, sin embargo este riesgo diminuye con el tratamiento con 
hidroxicloroquina, estos datos que coinciden con los de otros autores como Docherty et $\mathrm{al}^{7}$; Marta et $\mathrm{al}^{12}$; Wang et $\mathrm{al}^{13} \mathrm{y}$ Chinese Center for disease Control and Prevention ${ }^{18}$. El resto de marcadores analíticos no parecen influir significativamente en el desenlace de los pacientes con COVID-19 como han publicado previamente Ferrando et $\mathrm{al}^{14}$. La relación entre el tratamiento con corticoides y la mortalidad en nuestro estudio, podría explicarse porque se le administró este tratamiento a pacientes graves y críticos que fueron los que tuvieron un mayor índice de mortalidad.

El tratamiento con hidroxicloroquina, lopinavir/ritonavir actuaron como factores protectores frente a la mortalidad en nuestros pacientes. Aunque la OMS posteriormente suspendió los ensayos clínicos con hidroxicloroquina y lopinavir/ritonavir basándose en la poca o ninguna reducción de la mortalidad y en los efectos secundarios, hidroxicloroquina y lopinavir/ritonavir fueron los fármacos más utilizados en aquellos pacientes que sufrieron la enfermedad en los momentos iniciales de la pandemia en nuestro país. En la actualidad las recomendaciones se basan en el tratamiento con corticoides, remdesivir y tocilizumab, dependiendo de la gravedad del cuadro y de subpoblaciones que desarrollan una respuesta inflamatoria exagerada como publican Rivera et al ${ }^{21}$. En nuestros pacientes el tratamiento con tocilizumab no disminuyó el riesgo de mortalidad, pero su prescripción se llevó a cabo en un número muy reducido de pacientes.

Este estudio tiene diversos puntos fuertes. En primer lugar, este estudio aportó una descripción altamente detallada de los antecedentes personales, tratamientos y evolución de los pacientes con COVID-19 de los 342 paciente ingresados en el EOXI de Ourense. En segundo lugar, es un estudio retrospectivo que analiza la asociación entre la historia previa del paciente, analíticas y tratamientos durante el ingreso con la mortalidad y el ingreso en cuidados intensivos en nuestra población así como los factores que influyen positiva y negativamente.

Sin embargo, reconocemos ciertas limitaciones. En primer lugar, es plausible que, debido a la carga de trabajo de los facultativos participantes durante el periodo de estudio, algunos de los pacientes ingresados no hubieran sido incluidos en la base de datos. En segundo lugar, en el momento del análisis algún paciente no tenían resultado definitivo con respecto al alta o la muerte y, por tanto puede variar mínimamente.

\section{CONCLUSIONES}

\section{Respondiendo a los objetivos planteados:}

- Edad avanzada y el tratamiento con antiagregantes se relacionaron con un menor riesgo de ingreso en cuidados intensivos, mientras que el tratamiento con inhibidores de la aldosterona y el aumento de LDH y Dímero $D$, se relacionaron con un mayor riesgo de ingreso en cuidados intensivos.

- Edad >75 años, niveles elevados de LDH y el tratamiento con corticoides se relacionaron con una mayor mortalidad. El tratamiento con hidroxicloroquina se relacionó con una menor mortalidad.
CONFLICTO DE INTERESES Y FUENTES DE FINANCIACIÓN

Los autores de este artículo declaramos no tener ningún conflicto de intereses ni tampoco fuente de financiación.

The authors declare that there is no conflict of interest in this work.

Financiación: The authors declare that there were no external sources of study for the performance of this article.

Aspectos éticos: The authors declare that no data that allows identification of the patient appears in this article."

\section{BIBLIOGRAFÍA}

1. Caizheng Yu, Quing Lei, Wenkai Li, Xiong Wang, Wei Liu, Xionglin Fan. Clinical charcteristics , associated factors, and predicting COVID-19 mortality risk: a retrospective study in Wuhan, China. Am J Pre Med. 2020; 59: 168-175.

2. Trilla A. Un mundo, una salud: la epidemia por el nuevo coronavirus Covid-19. Med clin. 2020; 154: 175-177.

3. Shao A, Zhou Y, Tu S, Sheng J. A novel scoring system in mortality prediction of severe patients with COVID-19. Eclinicalmedicine. 2020;24: 1-2.

4. 2020-08-16_COVID19_Web_CifrasTotais. Dirección General de Salud Pública de la Consellería de Sanidad de la Xunta de Galicia.

5. Yan L, Hai-Tao Zhang, Goncalves J, Xiao Y, Wang M, Guo Y. An interpretable mortality prediction model for COVID-19 patients. Nature Machine Intelligence. 2020; 2: 283-288.

6. RichardsonS, HirschJS, NarasimhanM, CrawfordJM, McGinnT,Davidson KW, et al. Presenting characteristics, comorbidities, and outcomes among 5700 patients hospitalized with COVID-19 in the New York City Area. JAMA. 2020; 323: 20522059.

7. Casas-Rojo J.M., Antón-Santos M, Millán J, Lumbreras C, Ramos JM, Artero A. Características clínicas de los pacientes hospitalizados con COVID-19 en España: resultados del Registro SEMI-COVID-19. Rev Clin Esp. 2020; 220:480-494.

8. Zhou F., Yu T., Du R., Fan G., Liu Y., Liu Z. Clinical course and risk factors for mortality of adult inpatients with COVID-19 in Wuhan, China: a retrospective cohort study. Lancet. 2020;395:1054-1062.

9. Docherty A.B., Harrison E.M., Green C.A., Hardwick H.E., Pius R., Norman L. Features of 20 ? 133 UK patients in hospital with covid-19 using the ISARIC WHO Clinical Characterisation Protocol: prospective observational cohort study BMJ. 2020;369:m1985.

10. Young BE, Ong SWX, Kalimuddin S, Low JG, Tan SY, Loh J, et al. Epidemiologic Features and Clinical Course of Patients Infected With SARS-CoV-2 in Singapore. JAMA. 2020; 323: 1488-1494.

11. Kujawski SA, Wong KK, Collins JP, Epstein L, Killerby ME, Midgley CM, et al. First 12 patients with coronavirus disease 2019 (COVID-19) in the United States. MedRxiv. 2020:;20032896.

12. Marta J, Corroza-Laviñeta J, Ostolaza A. Factores de riesgo y predictores de gravedad en pacientes hospitalizados por Covid-19: análisis de 52 casos. Med Clin 2020; 155:360-361.

13. Wang D., Hu B., Hu C., Zhu F., Liu X., Zhang J. Clinical characteristics of 138 hospitalized patients with 2019 novel coronavirus-infected pneumonia in Wuhan, China. JAMA. 2020;323:1061-1069.

14. Ferrando C, Mellado-Artigas R, Gea A, Arruti E, Aldecoa C , Bordell A et al. Características, evolución clínica y factores asociados a la mortalidad en UCI de los pacientes críticos infectados por SARS-CoV-2 en España: estudio prospectivo, de cohorte y multicéntrico. Rev Esp Anestesiol Reanim. 2020; 67: 425-437..

15. Zhang Q, Bastard P, Liu Z , Le Pen J, Moncada-Velez M, Chen J et al. Inborn errors of type I IFN immunity in patients with life-threatening COVID-19. Science 2020;370:422-436.

16. Chen G., Wu D., Guo W., Cao Y., Huang D., Wang H. Clinical and immunologic features in severe and moderate coronavirus disease 2019. J Clin Invest. 2020; 130: 2620-2629.

17. Onder G., Rezza G., Brusaferro S. Case-fatality rate and characteristics of patients dying in relation to COVID-19 in Italy. JAMA. 2020;323:1775-1776

18. Rapid Risk Assessment. Coronavirus disease 2019 (COVID-19) in the EU/EEA and the UK - eighth update 2020. Disponible en: https://www.ecdc.europa.eu/en/ current-risk-assessment-novel-coronavirus-situation

19. Chinese Center for Disease Control and Prevention.

The Novel Coronavirus Pneumonia Emergency Response Epidemiology Team. CCDC Weekly. 2020;2: 113-123.

20. Shang Y, Liu T, Wei Y, Li J, Shao L, Liu M, et al. Scoring systems for predicting morality for severe patients with COVID-19. E Clin Med. 2020; 24:1000426.

21. Rivera M, Valero MC, R del Amo JL, Fernández MA, Martínez S, Tahery Arezu. Agentes terapéuticos utilizados en 238 pacientes hospitalizados por COVID-19 y su relación con la mortalidad. Med clin. 2020; 155:375-381. 